\title{
CONTROLE PRESSÓRICO E INÉRCIA TERAPÊUTICA NO AMBULATÓRIO ESCOLA DA UNIVERSIDADE IGUAÇU
}

\author{
PRESSURE CONTROL AND THERAPEUTIC INERTIA IN THE OUTPATIENT \\ SCHOOL OF THE IGUAÇU UNIVERSITY
}

Ana Cláudia Moraes Silva, ${ }^{1 *}$ Shanna silva mello, ${ }^{1 *}$ Pedro Costa Vital, ${ }^{1 *}$ Isabela Araujo Anuda, ${ }^{1 *}$ Rodrigo Nakamura Soares, ${ }^{1^{*}}$ Severino Veras de Oliveira Júnior, ${ }^{*}$ Caroline Torres Rodrigues, ${ }^{1,2,3}$ Roberta Rodrigues Teixeira de Castro, ${ }^{1,4}$ Rafael Costa, ${ }^{5}$ Jorge Ferreira, ${ }^{6,7,8}$ Erika Maria Gonçalves Campana ${ }^{1,9,10}$

\section{RESUMO}

Introdução: Conhecer as taxas de controle da pressão do Ambulatório Escola da Universidade Iguaçu, as estratégias terapêuticas e a taxa de inércia terapêutica frente aos pacientes não controlados, são fundamentais para estabelecer estratégias de alcance de metas pressóricas. Objetivo: Determinar a taxa de pacientes na meta e fora da meta de pressão arterial, a estratégia terapêutica em uso e a taxa de inércia terapêutica frente aos pacientes não controlados. Metodologia: Estudo observacional, prospectivo e analítico através de análise dos prontuários e entrevista com pacientes consecutivos atendidos no ambulatório escola da Universidade Iguaçu, com >18 anos, no período de agosto a dezembro de 2019. Análise de dados demográficos, condições clínicas associadas e presença de outros fatores de risco. Resultados: 202 pacientes estudados na pesquisa, faixa etária de 18 a 85 anos, com 91,08\% dos pacientes com hipertensão arterial (40 e 79 anos). Analisando as taxas de controle, 61,38 \% encontram-se na meta de pressão arterial de acordo com o risco cardiovascular, segundo a Sociedade Brasileira de Cardiologia, e 38,6\%, dos pacientes não se encontram na meta. A adesão terapêutica elevada foi observada em $29,83 \%$ dos pacientes na meta vrs $26,92 \%$ dos pacientes fora da meta. A taxa de inercia terapêutica foi de $41,03 \%$ pacientes com conduta mantida. Conclusão: Dos pacientes atendidos, 38,61\% estão fora da meta de pressão arterial, e a inercia terapêutica é um importante problema. Como a maioria dos pacientes hipertensos sem comorbidades são acompanhados nas unidades básicas de saúde por generalistas, a capacitação desses profissionais é de grande importância para um melhor controle da pressão arterial.

Descritores: Hipertensão arterial; Inércia Terapêutica; Pressão Arterial; Adesão Terapêutica.

\section{ABSTRACT}

Introduction:Knowing the pressure control rates of the outpatient school at the Iguaçu University, therapeutic strategies and the rate of therapeutic inertia in the face of uncontrolled patients are essential to establish strategies to achieve pressure goals. Objective: Determine the rate of patients on and off the blood pressure target, the therapeutic strategy in use and the rate of therapeutic inertia compared to uncontrolled patients. Methodology:Observational, prospective and analytical study through analysis of medical records and interviews with consecutive patients over 18 years old seen at the outpatient school at Universidade Iguaçu, from August to December 2019. Analysis of demographic data, associated clinical conditions and the presence of others risk factors. Results:202 patients studied in the research, age range from 18 to 85 years, with $91.08 \%$ of patients with arterial hypertension (40 and 79 years). Analyzing the control rates, $61.38 \%$ are on the blood pressure target according to cardiovascular risk, according to the Brazilian Society of Cardiology, and 38.6\%, of the patients are not on the target. High therapeutic adherence was observed in $29.83 \%$ of patients on target vs. $26.92 \%$ of patients off target. The

1. Universidade Iguaçu (UNIG), Nova Iguaçu, RJ, Brasil.

2. Hospital Quinta D'Or, São Cristóvão, Rio de Janeiro, RJ, Brasil.

3. Ambulatório Hospital Geral de Nova Iguaçu - Hospital da Posse (HGNI), Nova Iguaçu, RJ, Brasil.

4. Instituto Nacional de Câncer José Alencar Gomes da Silva (INCA).

5. Pesquisador Independente, Cardiologista e Ergometrista pela SBC/ AMB

6. Laboratório de Habilidades e Simulação, Universidade Iguaçu (UNIG), Nova Iguaçu, RJ, Brasil.

7. Unidade de Terapia Intensiva, Hospital Geral de Nova Iguaçu, Nova Iguaçu, RJ, Brasil.

8. Cardiologista da Prefeitura de Nova Iguaçu, Nova Iguaçu, RJ, Brasil.

9. Ambulatório de Hipertensão e Lípides, Departamento Cardiopulmonar, Universidade do Estado do Rio de Janeiro (UERJ), RJ, Brasil.

10. Núcleo Especializado de Hipertensão, Clínica SEACOR, RJ, Brasil.

${ }^{*}$ Graduando em medicina. 
therapeutic inertia rate was $41.3 \%$ of patients in the target. Conclusion: Of the patients seen, $38.61 \%$ are outside the blood pressure target, and therapeutic inertia is an important problem. As the majority of hypertensive patients without comorbidities are monitored in basic health units by general practitioners, the training of these professionals is of great importance for a better control of blood pressure.

Keywords: High Blood Pressure;Therapeutic Inertia; Blood Pressure; Treatment Adherence.

\section{INTRODUÇÃO}

A Hipertensão Arterial Sistêmica (HAS) é uma entidade clínica definida como níveis elevados e sustentados da pressão arterial (PA). Leva a disfunções funcionais e estruturais de órgãos alvo (vasos sanguíneos, rins, encéfalo e coração), além de alterações metabólicas, levando a um aumento expressivo do risco cardiovascular global de forma linear, contínua e independente em níveis de PA acima de 115/75mmHg. Possui alta prevalência e baixas taxas de controle, o que implica em grande morbimortalidade cardiovascular e altos custos financeiros. ${ }^{1}$

O impacto médico e social da HAS é enorme, ela encontra-se presente em $69 \%$ dos pacientes com primeiro episódio de infarto do miocárdio; $77 \%$ dos pacientes com acidente vascular cerebral; $75 \%$ dos pacientes com insuficiência cardíaca e $60 \%$ dos pacientes com DAP. Sendo responsável por $45 \%$ das mortes cardíacas e $51 \%$ das mortes decorrentes de AVE 1 . No Brasil, a HAS contribui direta ou indiretamente para $50 \%$ das mortes por doença cardiovascular (DCV), doença esta que representa 339.672 das causas de morte, sendo a principal causa de morte no país. Além disto, as DCV são responsáveis por uma alta frequência de internações, com custos socioeconômicos elevados. Ao longo dos anos de 2002 a 2009, as taxas de mortalidade CV têm apresentado redução, entretanto este padrão não foi observado em relação a doença hipertensiva, cujas taxas, no período, oscilaram de 39/100.000 habitantes (2000) para 42/100.000 habitantes, reforçando a importância do problema. ${ }^{1}$

Detectar, tratar e controlar níveis elevados da PA sabidamente reduz a ocorrência de eventos cardiovasculares. Na população brasileira as taxas de conhecimento, tratamento e controle variaram bastante, dependendo da população estudada. Uma revisão com estudos nacionais, mostrou as taxas de conhecimento (22\% a $77 \%)$, tratamento $(11,4 \%$ a $77,5 \%)$ e controle (10,1\%a 35,5\%) amplamente variáveis. ${ }^{1}$

Sabidamente a hipertensão arterial é uma doença multifatorial e os pacientes hipertensos habitualmente agregam outros fatores de risco cardiovascular (FR) e condições clínicas associadas que aumentem o risco de morbidade e mortalidade atribuível a doença hipertensiva. Identificar todos os elementos clínicos para definição do risco cardiovascular global do paciente é fundamental para estabelecermos uma estratégia terapêutica capaz de reduzir as complicações da hipertensão arterial. ${ }^{1}$ A hipertensão não controlada é extremamente comum em todo o mundo e, com base em dados epidemiológicos, é responsável por um grande número de mortes e invalidez. Efeitos negativos sobre as tendências decorrem, não somente da não-adesão ao tratamento, como do subtratamento, inadequação da droga, dificuldade do acesso ao sistema de saúde, indisponibilidade de medicação na rede básica de saúde, quantidade de drogas e número de doses diárias da medicação prescrita, efeitos adversos, resistência ao tratamento e presença de comorbidades. ${ }^{2,3}$

A proposta do presente estudo é de estabelecer a prevalência de HAS, identificar o perfil epidemiológico e de risco cardiovascular dos pacientes portadores de HAS, determinar a taxa de controle da pressão arterial e identificar os fatores que dificultem o alcance das metas terapêuticas, com objetivo de propor estratégias modificadoras de tais fatores, sendo priorizada a prevenção da inércia terapêutica frente aos pacientes não controlados, juntamente com outras medidas importantes para controlar a hipertensão e minimizar suas consequências.

\section{MATERIAL E MÉTODOS}

Todos os participantes do estudo foram esclarecidos sobre o estudo e assinaram o termo de consentimento livre esclarecido. O artigo foi submetido ao comitê de ética UNIG com o número de protocolo CAAE:13972719.9.0000.8044.

Estudo de caráter observacional, prospectivo e analítico através de análise dos prontuários e entrevista com os pacientes assistidos pelo ambulatório escola da Universidade de Nova Iguaçu (CMS Vasco Barcelos). Os critérios de inclusão são a idade maior que 18 anos e ser atendido em consulta no ambulatório escola de cardiologia da Universidade Nova Iguaçu. A coleta de dados iniciou em 01/10/2019, incluindo todos os pacientes consecutivos atendidos nos ambulatórios de cardiologia.

Os dados foram coletados por meio de um questionário e os pacientes analisados através de dados demográficos, da condição atual da, das condições clínicas associadas e analisada a presença de outros fatores de risco cardiovascular.

A PA foi medida no membro superior de maior valor de pressão, de acordo com as recomendações das VII Diretrizes Brasileiras de Hipertensão ${ }^{1}$. Sendo realizados dois registros da PA, com intervalos de dois minutos entre cada medida, sendo a média delas utilizada para análise. A classificação da hipertensão, a estratificação de risco cardiovascular e meta de PA foi classificada de acordo com as recomendações das VII Diretrizes Brasileiras de Hipertensão (R1). ${ }^{1}$

Índice de massa corporal, calculado pela formula: $I M C=P / A^{2}$; circunferência abdominal, medida com trena antropométrica portátil inelástica de polietileno sendo medida no ponto médio entre a borda inferior da última costela e a crista ilíaca.

A avaliação da aderência terapêutica, realizada pelo Teste de Morisky-Green ${ }^{4}$ sendo considerados os seguintes resultados: Baixa adesão (0 a $<6$ respostas positivas); Moderada Adesão (6 a $<8)$; Alta adesão (8 respostas positivas).

A Pesquisa de suspeita de apneia obstrutiva do sono foi realizada através dos questionários de Berlim e através da medida do pescoço, realizada com trena antropométrica portátil inelástica de polietileno sendo medida exatamente 
no meio da distância da altura, ou tendo como referência a proeminência laríngea e a conduta terapêutica adotada pelo médico assistente foi avaliada por um questionário de respostas possíveis (Figura 1 ).

\section{RESULTADOS}

Dos 223 pacientes convidados para a pesquisa, 202 concordaram em participar. As características demográficas da população estudada estão expostas na tabela 1. Na tabela 2 estão descritos os fatores de risco adicionais nos pacientes hipertensos; quase $1 / 3$ da população estudada apresenta diabetes mellitus e obesidade. O número de diabéticos pode ser ainda maior, muitos pacientes não sabiam informar ou não tinham realizado exames laboratoriais de glicemia de jejum e/ou HbA1C (hemoglobina glicada). Mais da metade confirmaram história familiar prematura de DVC em parentes de $1^{\circ}$ grau e sedentarismo. Não foi realizada a pesquisa das lesões subclínicas de órgãos alvo, entretanto na análise de prontuários, encontramos 10 pacientes com HVE, 01 com espessamento médio intimal de carótidas $>0,9 \mathrm{~mm}$; $01 \mathrm{com}$ VOP $>10 \mathrm{~m} / \mathrm{s} ; 2$ pacientes com albuminuria $>30 \mathrm{mg} / 24 \mathrm{~h}$. A presença de doenças concomitantes relacionadas a morbidade da hipertensão foi obtida por questionário com a informação do paciente não sendo realizado verificação de documentação da ocorrência dos eventos CV. (Tabela 3)

Após o conhecimento dos FR, lesões de órgãos alvo e doenças associadas, pudemos avaliar o estadiamento do risco cardiovascular desses pacientes, encontramos 39,6\% dos pacientes com alto RCV; 30,2\% com RCV moderado e $30,2 \%$ com baixo RCV, ou seja, a maior parte dos pacientes

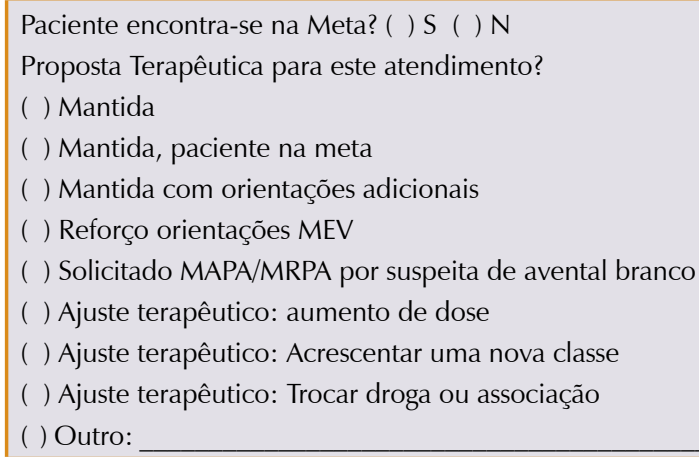

Figura 1. Avaliação da conduta terapêutica adotada pelo médico assistente em pacientes hipertensos que se encontram na meta ou não.

Tabela 1. Características demográficas da população estudada.

\begin{tabular}{l|l}
\multicolumn{1}{c|}{ Características } & \multicolumn{1}{|c}{ Geral $(\mathbf{n}=202)$} \\
\hline $\begin{array}{l}\text { Idade em anos (média } \pm \text { desvio } \\
\text { padrão) }\end{array}$ & $56,94 \pm 9,30$ \\
\hline Sexo (M / F) - n (\%) & $65,4 \%(132) / 34,6 \%(70)$ \\
\hline $\begin{array}{l}\text { Cor (Branco / Preto / Não branco e } \\
\text { não preto) }\end{array}$ & $\begin{array}{l}41,6 \%(84) / 25,7 \%(52) / \\
32,6 \%(66)\end{array}$ \\
\hline $\begin{array}{l}\text { Estado civil (com companheiro / sem } \\
\text { companheiro) - } \mathrm{n}(\%)\end{array}$ & $53,5 \%(108) / 36,5 \%(94)$ \\
\hline $\begin{array}{l}\text { Educação (Analfabeto / Alfabetizado / } \\
\text { Ensino fundamental / Ensino médio e } \\
\text { Ensino superior) - } \mathrm{n}(\%)\end{array}$ & $\begin{array}{l}11,4 \%(23) / 16,3 \%(33) \\
\text { (4) }\end{array}$ \\
\hline
\end{tabular}

Tabela 2. Fatores de risco cardiovascular na avaliação do risco adicional no hipertenso.

\begin{tabular}{|c|c|}
\hline Características & Geral $(n=202)$ \\
\hline Fumante (sim / não) - n (\%) & 9,4\% (19) / 90,59\% (183) \\
\hline Uso de álcool (sim / não) - n (\%) & $13,86 \%(28) / 86,3 \%(174)$ \\
\hline $\begin{array}{l}\text { Atividade física (ativa / sedentária) - } \\
\mathrm{n}(\%)\end{array}$ & $48,51 \%(98) / 51,48 \%(104)$ \\
\hline $\begin{array}{l}\text { Diabetes (sim / não ou não sabem) - } \mathrm{n} \\
(\%)\end{array}$ & $24,75 \%(50) / 75,25 \%(152)$ \\
\hline $\begin{array}{l}\text { Excesso de Peso }\left(\mathrm{IMC} \geq 30 \mathrm{~kg} / \mathrm{m}^{2}\right. \\
\text { ou } \mathrm{CA} \geq 102 \mathrm{~cm} \text { nos homens ou } \\
\geq 88 \mathrm{~cm} \text { nas mulheres) (sim / não) } \\
-\mathrm{n}(\%)\end{array}$ & $24,75 \%(50) / 75,25 \%(152)$ \\
\hline $\begin{array}{l}\text { História familiar prematura de DCV } \\
\text { em parentes } 10 \text { Grau: }(\mathrm{H}<55 ; \mathrm{M}< \\
65) \text { (Sim / Não ou não sabem) - N } \\
(\%)\end{array}$ & $56,4 \% \%(114) / 43,6 \%(88)$ \\
\hline $\begin{array}{l}\text { Dislipidemia (Colesterol total } \\
>190 \mathrm{mg} / \mathrm{dl} \text { e/ou LDL-colesterol } \\
>115 \mathrm{mg} / \mathrm{dl} \text { e/ou HDL-colesterol } \\
<40 \mathrm{mg} / \mathrm{dl} \text { nos homens ou } \\
<46 \mathrm{mg} / \mathrm{dl} \text { nas mulheres e/ou } \\
\text { Triglicerídeos > } 150 \mathrm{mg} / \mathrm{dl} \text { ) } \\
\text { (Sim / Não ou Não sabem }\end{array}$ & $29,2 \%(59) / 70,8 \%(143)$ \\
\hline $\begin{array}{l}\text { Hiperuricemia: (Sim / Não ou Não } \\
\text { sabem) }\end{array}$ & $5,44 \%(11) / 94,5 \%(191)$ \\
\hline
\end{tabular}

Tabela 3. Doença CV e renal estabelecida para avaliação do risco adicional no hipertenso.

\begin{tabular}{|c|c|}
\hline Características & Geral $(n=202)$ \\
\hline $\begin{array}{l}\text { Doença Arterial Obstrutiva } \\
\text { Periférica Assintomática ou Índice } \\
\text { tornozelo-braquial <0,9: } \\
\text { (Sim / Não ou Não sabem) }\end{array}$ & $9,4 \%(19) / 90,59 \%(183)$ \\
\hline $\begin{array}{l}\text { Doença Renal Crônica Estagio } 3 \\
\text { (TFG CKD-EPI: } 30-60 \mathrm{ml} / \mathrm{min} / \\
\text { 1,73m²): (Sim / Não ou Não } \\
\text { sabem) }\end{array}$ & $13,86 \%$ (28) / 86,3\% (174) \\
\hline $\begin{array}{l}\text { Doença cerebrovascular } \\
\text { (AVE isquêmico e/ou Hemorragia } \\
\text { cerebral e/ou Ataque isquêmico } \\
\text { transitório prévios) - (Sim / Não ou } \\
\text { Não sabem) }\end{array}$ & $48,51 \%(98) / 51,48 \%(104)$ \\
\hline $\begin{array}{l}\text { Doença da artéria coronária } \\
\text { (Angina Estável ou Angina Instável } \\
\text { ou IAM ou Angioplastia ou CRVM } \\
\text { prévios): (Sim / Não ou Não } \\
\text { sabem) }\end{array}$ & $24,75 \%(50) / 75,25 \%(152)$ \\
\hline $\begin{array}{l}\text { Doença Arterial Obstrutiva } \\
\text { Periférica Sintomática } \\
\text { (Claudicação): (Sim / Não ou Não } \\
\text { sabem) }\end{array}$ & $24,75 \%(50) / 75,25 \%(152)$ \\
\hline $\begin{array}{l}\text { Doença renal crônica estágio } \\
4 \text { (TFG CKD-EPI: < } 30 \mathrm{ml} / \\
\text { min/ } 1,73 \mathrm{~m}^{2} \text { ou albuminúria } \\
>300 \mathrm{mg} / 24 \text { h): } \\
\text { (Sim / Não ou Não sabem) }\end{array}$ & $56,4 \% \%(114) / 43,6 \%$ (88) \\
\hline $\begin{array}{l}\text { Retinopatia Avançada } \\
\text { (hemorragias, exsudatos, } \\
\text { papiledema): (Sim / Não ou Não } \\
\text { sabem) }\end{array}$ & $29,2 \%$ (59) / 70,8\% (143) \\
\hline
\end{tabular}


estudados apresentam alto risco cardiovascular, isso se dá pelo acúmulo de múltiplos fatores de riscos.

Analisando as taxas de controle da pressão arterial, 61,38 \% encontram-se na meta de pressão arterial de acordo com o risco cardiovascular, segundo a VII Diretriz Brasileira de Hipertensão Arterial $^{1}$. Mais de $1 / 3$ dos pacientes estavam fora da meta de pressão arterial. Na tabela 4 e 5 respectivamente avaliamos a estratégicas terapêuticas nos pacientes que se encontram na meta ou fora da meta pressórica com relação ao uso de fármacos. A pesquisa mostrou que quanto mais classes de fármacos prescritos e maior o número de comprimidos a serem ingeridos várias vezes ao dia, pior é a aderência terapêutica. Na tabela 6 observamos que o número de pacientes com elevada adesão terapêutica que se encontram na meta pressórica, foi de 29,83\% e os pacientes com elevada adesão terapêutica que não se encontram na meta pressórica foi de $26,82 \%$. Com relação a Taxa de Inércia Terapêutica, demobservamos que $41 \%$ dos pacientes em tratamento para hipertensão arterial estavam fora da meta pressórica, porém não foi realizado ajuste terapêutico por parte dos profissionais médicos. (Tabela 7)

\section{DISCUSSÃO}

A taxa de sedentarismo entre os pacientes hipertensos estudados na amostra é de mais 50\%, contribuindo também para a taxa de excesso de peso que se mostrou bastante expressiva, agravando a condição de saúde desses pacientes.

Tabela 4. Estratégia terapêutica dos pacientes que se encontram na meta pressórica.

\begin{tabular}{l|c}
\multicolumn{1}{c|}{ Características } & $\begin{array}{c}\text { Pacientes na meta } \\
\mathrm{n}=124\end{array}$ \\
\hline Mudança estilho de vida isoladamente & $12,09 \%(15)$ \\
\hline Monoterapia & $20,16 \%(26)$ \\
\hline 2 Fármacos & $22,58 \%(28)$ \\
\hline 3 Fármacos & $19,35(24)$ \\
\hline Mais de 3 fármacos & $16,93(21)$ \\
\hline Não fazem tratamento & $5,44 \%(11)$ \\
\hline
\end{tabular}

Tabela 5. Estratégia terapêutica dos pacientes que não se encontram na meta pressórica.

\begin{tabular}{l|c}
\multicolumn{1}{c|}{ Características } & $\begin{array}{c}\text { Pacientes fora da meta } \\
\mathbf{n = 7 8}\end{array}$ \\
\hline Mudança estilho de vida isoladamente & $6,41 \%(5)$ \\
\hline Monoterapia & $16,66 \%(13)$ \\
\hline 2 fármacos & $29,48 \%(23)$ \\
\hline 3 fármacos & $15,38 \%(12)$ \\
\hline Mais de 3 fármacos & $28,20 \%(22)$ \\
\hline Não fazem tratamento & $1,48 \%(3)$ \\
\hline
\end{tabular}

Tabela 6. Adesão terapêutica segundo questionário de Morisk-Green*

\begin{tabular}{|c|c|}
\hline Características & \\
\hline $\begin{array}{l}\text { Adesão terapêutica elevada* dos pacientes na } \\
\text { meta } n=124\end{array}$ & $29,83 \%(37)$ \\
\hline $\begin{array}{l}\text { Adesão terapêutica elevada* pacientes fora da } \\
\text { meta } N=78\end{array}$ & $26,92 \%(21)$ \\
\hline
\end{tabular}

*Questionário de Morisky-Green é uma ferramenta útil na avaliação da adesão terapêutica do paciente e consta como resultados: Baixa adesão $(0$ a $<6$ respostas positivas); Moderada adesão ( 6 a $<8$ respostas positivas); Adesão elevada ( 8 respostas positivas).
Tabela 7. Inércia terapêutica.

\begin{tabular}{l|c}
\multicolumn{1}{c|}{ Características } & $\begin{array}{c}\text { Pacientes fora da } \\
\text { meta } \mathbf{n = 7 8}(38,6 \%)\end{array}$ \\
\hline $\begin{array}{l}\text { Taxa de inercia terapeutica com conduta } \\
\text { mantida }\end{array}$ & $25,65 \%(20)$ \\
\hline $\begin{array}{l}\text { Taxa de inercia terapêutica com conduta } \\
\text { mantida com orientação }\end{array}$ & $15,38 \%(12)$ \\
\hline Modificação da estratégia terapêutica & $58,97 \%(46)$ \\
\hline
\end{tabular}

A literatura destaca o sedentarismo como um dos principais fatores de risco para doenças cardiovasculares. Concordante com nossos resultados, um estudo realizado com hipertensos no nordeste do Brasil, o estilo de vida sedentário foi identificado em $55,8 \%$ dos participantes. ${ }^{5}$

Segundo a VII diretriz de hipertensão arterial, ${ }^{1}$ associação de HA e DM dobra o risco CV e aumenta a prevalência de HA. ${ }^{1}$ Quase $25 \%$ dos hipertensos estudados tem o diagnóstico de DM e os demais assistidos da amostra não são diabéticos ou não sabem. Outros estudos também demonstram valores parecidos ou maiores de hipertensos portadores de diabetes concomitantemente. ${ }^{6,7,8}$

Hipertensos com história familiar de DCV prematura em parentes de primeiro grau apresentam um risco adicional elevado de sofrerem um evento cardiovascular (Homens < 55 anos ou mulheres < 65 anos). ${ }^{1}$ Nosso estudo mostrou que mais da metade dos hipertensos estudados referem história familiar prematura de DCV, mas como nós não tivemos acesso aos prontuários dos familiares, este dado representa uma limitação do estudo, pois não foi confirmado por documentação do evento.

A maioria dos pacientes da amostra apresentaram como estadiamento de risco $\mathrm{CV}$ a classificação de alto risco, comparados aos números de pacientes com risco moderado e baixo risco CV. Essa situação se justifica pelo grande número de pacientes hipertensos que acumulam vários fatores de riscos adicionais, como diabetes, sedentarismo, obesidade, tabagismo, lesões de órgãos alvos (hipertrofia ventricular esquerda, insuficiência renal crônica, acidente vascular encefálico, insuficiência cardíaca, etc).

Com relação as estratégias terapêuticas nos pacientes que se encontram na meta pressórica, fica claro que quanto menor o número de classes de fármacos e de comprimidos a serem ingeridos ao dia, melhor a adesão terapêutica, contribuindo para uma melhor resposta frente ao tratamento realizado. A adesão pode ser melhorada com a prescrição de farmacoterapia combinada em dose fixa, porém o SUS não disponibiliza para tratamento e controle da hipertensão arterial, farmacoterapia combinada de 2 ou mais tipos de fármacos em apenas um comprimido, o que facilitaria a adesão terapêutica, sinergismo farmacólogo e consequentemente um aumento significativo da taxa de controle dos hipertensos. A terapia combinada de dose fixa com duas classes diferentes de agentes anti-hipertensivos alcançará pressões de meta em mais de $70 \%{ }^{9}$

Uma metanálise realizada com artigos que foram recuperados dos bancos de dados MEDLINE e Embase usando uma combinação de termos "combinações de doses fixas" e "adesão ou adesão ou persistência" e "hipertensão ou anti-hipertensivo" 
de janeiro de 2000 a junho de 2017 sem nenhuma restrição de idioma, confirma que as terapias de combinações de doses fixas ( CDFs), comparadas às combinações de equivalentes livres, estão associadas a uma melhor adesão ou persistência à medicação em pacientes com hipertensão. É razoável que médicos, farmacêuticos e formuladores de políticas facilitem o uso de CDFs para pacientes que precisam tomar dois ou mais medicamentos anti-hipertensivos. ${ }^{8}$

Comparando a alta adesão terapêutica nos grupos de pacientes fora da meta pressórica versus pacientes dentro da meta, observamos um discreto aumento de pacientes na meta com alta aderência terapêutica comparado ao grupo de pacientes fora da meta com alta adesão terapêutica. Essa diferença deveria ser bem maior, uma vez comprovada em estudos que quanto maior a aderência terapêutica, maior a taxa de controle. Esse cenário pode ser compreendido em consequência da alta taxa de inercia terapêutica analisada na pesquisa. Atualmente tem se estudado muito a questão da inércia terapêutica como forma de se comprovar que muitos casos de hipertensão arterial diagnosticada como de difícil controle ou não controlada, pode ser justificada pelo não tratamento adequado realizado pelo profissional médico, frente a conduta terapêutica adequada e a falta de orientação dada ao paciente.

O número de pacientes fora da meta com conduta terapêutica mantida e conduta mantida com orientação foi superior a $40 \%$, ou seja, esses pacientes não tiveram alteração da conduta terapêutica pelos médicos assistentes mesmo estando fora da meta. No Registro Brasileiro de Hipertensão a taxa de controle de pacientes quando se considerava a meta mais agressiva da pressão arterial $(<130 / 80 \mathrm{mmHg})$ foi de $24,3 \%$ sinalizando para um elevado número de pacientes com controle pressórico insatisfatório em nosso país. ${ }^{10}$ A inércia terapêutica é um grande problema de saúde pública, visto que os profissionais médicos, principalmente na atenção primária, apresentam grandes dificuldades no tratamento e controle da hipertensão arterial. Essa questão merece atenção especial dos profissionais de saúde, principalmente dos médicos que atuam junto à população hipertensa, já que tais variáveis mostraram associação com inadequado tratamento terapêutico no controle da hipertensão arterial.

A revisão da literatura mundial sugere que programas de educação continuada que utilizam múltiplas ferramentas de ensino e treinamento, adaptadas às condições locais, e que intervêm sobre estudantes, residentes, médicos e outros profissionais da saúde têm possibilidade de sucesso. O acesso facilitado aos médicos especialistas, o atendimento multiprofissional e as intervenções de ordem administrativa também são importantes para melhorar o controle da doença. ${ }^{10}$

\section{CONSIDERAÇÕES FINAIS}

A prevalência da hipertensão arterial sistêmica (HAS) é crescente no mundo e é o principal fator de risco para as doenças cardiovasculares e pelo impacto na qualidade de vida da população e do sistema de saúde. Apesar de existirem tratamentos eficazes, as taxas de descontrole da doença oscilam de 35\% a 80\%. A baixa adesão ao tratamento está presente em 50\% dos casos de pacientes hipertensos descompensados. A inércia terapêutica, também é um impedimento considerável para o controle adequado da hipertensão, o que tem implicações no prognóstico da doença. Mais de $38 \%$ dos pacientes atendidos no nosso ambulatório escola estão fora da meta de pressão arterial, e a inercia terapêutica é um importante problema. Como a maioria dos pacientes hipertensos sem comorbidades são acompanhados nas unidades básicas de saúde por generalistas, a capacitação desses profissionais é de grande importância para um melhor controle da pressão arterial.

\section{REFERÊNCIAS}

1. Malachias MVB, Plavnik FL, Machado CA, Malta D, Scala LCN, Fuchs S, Sociedade Brasileira de Cardiologia. 7a Diretriz Brasileira De Hipertensão Arterial. Arq Bras Cardiol. 2016;107(3):1-83.

2. Lessa I. Impacto social da não-adesão ao tratamento da hipertensão. Rev Bras Hipertens. 2006; 13(1): 39-46.

3. Rea F, Corrao G, Merlino L, Mancia G. Early cardiovascular protection by initial two-drug fixed-dose combination treatment vs. monotherapy in hypertension. Eur Heart J. 2018;39(40):3654-61.

4. Ben AJ, Neumann CR, Mengue SS. Teste de Morisky-Green e Brief Medication Questionnaire para avaliar adesão a medicamentos. Rev Saúde Pública. 2012;46(2):279-89.

5. Martins LCG, Lopes MVO, Guedes NG, Nunes MM, Diniz CM, Carvalho PMO. Sedentary lifestyle in individuals with hypertension. Rev Bras Enferm. 2015;68(6):697-704.

6. Guimarães Filho GC, Sousa ALL, Jardim TSV, Souza WSB, Jardim

PCVB. Progressão da pressão arterial e resultados cardiovasculares em pacientes hipertensos em um centro de referência. Arq Bras Cardiol. 2015 abr; 104 (4): 292-298

7. Girotto E, Andrade SM, Cabrera MAS, Ridão EG. Prevalência de fatores de risco para doenças cardiovasculares em hipertensos registrados na unidade de saúde da família. Acta Scientiarum. Ciências da Saúde. 2009; 31(1): 77-82.

8. Novello MF, Rosa MLG, Ferreira RT, Nunes IG,2 Jorge AJL,1 Correia DMS, et al. Conformidade da Prescrição Anti-Hipertensiva e Controle da Pressão Arterial na Atenção Básica. Arq Bras Cardiol. 2017; 108(2):135-142.

9. Moser M, Black HR. O papel da terapia combinada no tratamento da hipertensão. American Journal of Hypertension. 1998; 5(11):73S-78S.

10. Lopes RD, Barroso WKS, Brandao AA, Barbosa ECD, Malachias MVB, Gomes $\mathrm{MM}$, et al; RBH Investigators. The First Brazilian Registry of Hypertension. Am Heart J. 2018;205:154-57. 